\title{
Les mesures de “Reconstruction-Réconciliation" vis-à-vis la paix durable au Rwanda $^{1}$ \\ Measures of Reconstruction-Reconciliation towards a lasting peace in Rwanda
}

\section{Maria Eugenia Rodrigues Trombini ${ }^{2}$}

\section{RÉSUMÉ}

Les politiques de reconstruction et réconciliation au Rwanda sont racontées d'une multitude de perspectives. Au landemain du conflit la paix à court terme était associée à la sécurité plutôt qu'à la démocratie. Même si la démocratie est un processus lent que doivent être incorporé par la societé civile, une analyse du discours pour l'unité nationale indiquent l'instrumentalisation du génocide et la distance entre cette paix positive et la violence structurelle de ce modèle de réconciliation à sens unique menée par le FPR.

Palavras-chave : politiques de paix, reconstruction, réconciliation, génocide

\begin{abstract}
The policies of reconstruction and reconciliation in Rwanda are narrated from several perspectives. Immediately after the conflict, peacebuilding meant a short-term peace associated to security rather than to democracy. Even though democracy is a gradual process that must be assimilated by the civil society, an analysis of the national unity argument suggests the instrumentalisation of genocide and the distance between ideal positive peace and this model of one way reconciliation carried out by the RPF.
\end{abstract}

Key-words : peace policies, reconstruction, reconciliation, genocide

"Je ne crois pas ceux qui disent qu'on a touché le pire de l'atrocité pour la dernière fois. Quand il y a eu un génocide, il peut y en avoir un autre, n'importe où, au Rwanda ou ailleurs; si la cause est toujours là et qu'on ne la connait pas. »

\footnotetext{
${ }^{1}$ Artigo recebido em 21 de fevereiro de 2014 e aprovado em 31 de março de 2014.

${ }^{2}$ Graduanda em Ciência Política, UFPR, Curitiba, Brasil
}

Conjuntura Global, Vol.3, n.1, jan./mar., 2014, p. 65-77. 
(Jeannette Ayinkamiye, survivant du génocide) 
De même qu'il ne peut y avoir « une seule » cause du génocide, parce qu'il est plutôt un « processus de bascule, complexe, imbriquant des dynamiques collectives et individuelles, de nature politique, sociale, psychologique, etc.» ${ }^{3}$ pour appréhender les politiques de paix au Rwanda depuis le génocide et, encore plus, les évaluer, il faut considérer plusieurs aspects. Pour une meilleure compréhension des politiques de paix après le génocide, il faut séparer les éléments à court terme des éléments à long terme. Dès lors que le but d'empêcher une guerre est beaucoup plus important au lendemain d'un conflit, on n'utilisera pas le concept de paix négative comme l'absence de violence ou la probabilité extrêmement faible de la reprise de la guerre ici, même si on parlera des résultats produits par la stabilité créée par ce modèle de paix. ${ }^{4}$ Les indicateurs qualitatifs minimums de paix, c'est-à-dire les pratiques et les institutions pour la résolution non violente des conflits, sont établis, sont stables et sont en cours de fonctionnement depuis l'administration du FPR, nous allons démontrer à partir des explications historiques comment la paix à court terme était associée à la sécurité plutôt qu'à la démocratie dans le cas du Rwanda.

Différemment, la définition de paix expansive ou positive exige a) l'égalité sociale, raciale et économique ; b) l'harmonie et la coopération entre les groupes ; et c) des institutions démocratiques libérales. C'est à partir de cette perspective qu'on discutera la relation entre la paix positive et la violence structurelle par rapport à l'ingénierie sociale du FPR et le but d'unité nationale. Dans la définition de violence structurelle, l'inégalité sociale est systématique et produit des effets négatifs sur un groupe de personnes. Selon ce contexte, proposé par Galtung, l'absence de violence interpersonnelle systématique constituerait la paix négative et l'absence de violence structurelle constituerait la paix positive. On voit bien que les deux types de violence doivent être évités par les politiques de paix, néanmoins dans le cas étudié, la paix négative suffit à expliquer dans quelle mesure les politiques implantées à court terme au Rwanda ont réussi à éliminer la violence interpersonnelle massive, politique. 5 Par conséquent, vis-à-vis de la situation actuelle, la définition de paix positive est plus

\footnotetext{
${ }^{3}$ Sémelin J., Pour sortir de la violence, Paris, Les Editions ouvrières, 1983, p.16

4 Truth-Seeking, Truth-Telling, and. Postconflict Peacebuilding: Curb the Enthusiasm?, International Studies Review, Vol. 6, No. 3, September 2004, p. 371

${ }^{5}$ Ici on considère les politiques de paix implantées à court-terme mais leur résultat, d'avoir réussi a êchaper la violence interpersonnelle, n'était pas atteint en 1996-1997 quand le FPR a mener des graves violations contre les hutus, mais à partir de ça.
} 
pertinente et sa relation avec l'existence de violence structurelle au sein de la société rwandaise sera discutée en détail. De prime abord, une partie sera consacrée à l'ingénierie instituionnelle menée par le FPR en ce qui concerne le contrôle politique de la société civile et puis une deuxième partie sur l'ingénierie sociale par rapport à l'unité nationale et l'histoire officielle.

Bien évidemment, le succès du gouvernement rwandais en matière de stabilité politique, développement économique, proportion élevée des femmes au Parlement, amélioration de l'éducation et du système de santé sont nécessaires pour empêcher la récurrence de violence à grande échelle. ${ }^{6}$ Les tentatives également d'améliorer la prestation de la justice et l'abolition de la peine de mort démontrent les efforts du FPR malgré la difficulté évidente de gouverner un État après un génocide. Cependant, ceux qui considèrent le Rwanda un succès remarquable et un exemple de reconstruction post-conflit aident à établir une image superficielle, restreinte à l'absence de conflit, de la société rwandaise contemporaine, qui est, comme nous le verrons, beaucoup plus complexe.

\section{La force de l'État rwandais face à la faiblesse de la société civile}

Malgré la reconnaissance de la nécessité d'une transition gérée dans les contextes post-génocidaire, le cas du Rwanda révèle la crainte que cette nécessité soit utilisée pour dissimuler une tentative d'assurer la mainmise par le FPR du pouvoir politique. ${ }^{7}$ Néanmoins, la proposition d'une paix durable démocratique est toujours en conflit avec l'autonomie et la souveraineté du pays, et la question de la démocratie doit être remise en contexte. Quand la violence génocidaire a pris fin en 1994, l'État rwandais était détruit et ruiné, mais pour faire une comparaison avec le Rwanda contemporain, ce n'est pas le seul point focal qui doit être pris en compte, on doit quand même considérer la période pré-coloniale, coloniale et post-indépendance. ${ }^{8}$

Dans cette perspective, la présence d'un État fort et centralisé associé au contrôle social a été une caractéristique constante des régimes rwandais à travers le temps. ${ }^{9}$ Le régime Habyarimana était un régime autoritaire quand en 1978 la nouvelle

\footnotetext{
${ }^{6}$ Straus, Scott, Waldorf, Lars, Remaking Rwanda : State-Building and Human Rights after Mass Violence, The University of Wisconsin Press, Wisconsin, 2011, p.7

72002 USAID Conflict Vulnerability Assessment of Rwanda

8 Waldorf, Lars, Straus, Scott...op. cit. p.14

9 ibid. p.14
} 
constitution a formalisé l'institution d'un Etat à parti unique, sans opposition et sans liberté de la presse. Pendant les accords de paix Arusha, après l'invasion du FPR, une réalité politique plus complexe et démocratique a été introduite, mais ça s'est passé entre 1992 et 1993 et le génocide a commencé l'année suivante. Bref, le dialogue n'existait pas, les institutions n'étaient pas fondées sur un contrat social opérant et, en conséquence, une culture civique n'était pas présente. L'état était plutôt une « extension des groupes sectionnels ou d'intérêts » ${ }^{10}$ et la société civile était loin de contrebalancer le pouvoir étatique pendant la période avant 1994. En fait, les institutions étatiques fortes de contrôle et mobilisation du travail ont été cruciales pour la nature rapide du génocide rwandais. ${ }^{11}$ Ça nous amène à la problématique suivante : Sachant que la démocratie est un processus lent, dans quelle mesure est-il possible d'espérer et juger un système politique qui n'a jamais existé avant le génocide dans la forme de culture déjà installée et appropriée par le peuple?

En plus, la violence du génocide a détruit l'infrastructure, discredité les institutions politiques et religieuses et dévasté les engagements de confiance sociale. ${ }^{12}$ L'état rwandais était abîmé en juillet 1994 quand le FPR est arrivé , et la peur de la démocratie multipartite, de la liberté d'expression, de la liberté de la presse se justifia puisque la protection de la sécurité était le but principal du gouvernement. Le procureur général Ngoga dans un entretien pour le magazine Time en 2010 a argumenté que ce sentiment est toujours là : «Il y a des gens qui, donné l'occasion, le feraient encore une fois. Il y a des politiques qui aimeraient que cela arrive. Il y a des groupes armés qui le veulent. Il y a une population qu'on continue à éduquer mais qui n'est pas assez éduqué dans la mesure où ils ne seront plus manipulés. „13 Ça veut dire que la liberté d'expression autours des crimes du FPR commis en 1994 -ces qui étaient racontés par Carla Del Ponte au TPIR-14 ne sera pas permise- au moins pas avant que la population soit suffisamment rééduquée. Et bien, que l'argument qui légitime cette approche est la

\footnotetext{
10 J Adakanye, "Rwanda/Burundi: "uni-ethnic" dominance and the cycles of armed ethnic formations", Social Identities:Journal for the Study of Race, Nation and Culture, 2 (1), 1996, p. 39 In. Silva-Leander p. 1605

11 Straus 2006, Verwimp 2006

12 Waldorf, Lars, Straus, Scot, op cit. p. 13

13 Waldorf, Lars, L'Instrumentalisation du Génocide : The RPF's campaign against « Genocide Ideology » In. Remaking Rwanda : State Building and Human Rights after Mass Violence, p. 58

${ }^{14}$ Hirondelle News Agency "Carla Del Ponte tells of her attempts to investigate RPF in her new book" 2 avril 2008.
} 
sécurisation de l'État rwandais et le but de veiller à ce que la stabilité règne, donc un interêt politique. ${ }^{15}$

Avant le génocide de 1994 la société civile n'avait aucune influence dans les politiques gouvernementales. Le régime de Paul Kagame a substitué le modus operandi précédent pour un autre dans lequel le "vous êtes avec nous, ou vous êtes contre nous" national prédomine. À cause de l'intimidation et la disparition des critiques au FPR l'individu craint et ne conteste pas les politiques gouvernamentales, incertain sur les conséquences. Les conditions pour soutenir le débat public ne sont pas présentes. Reyntjens propose que la société civile rwandaise soit controlée par le régime, mais selon Paul Gready des espaces éventuels existent, cependant ils sont ad hoc et personalisés, plutôt que basés dans les relations institutionnelles entre la société et l'état. Il prend l'exemple des ONGs, nationales et internationales, qui depuis avril 2001 ont sa gestion, ses finances et ses projets sous contrôle gouvernamental. Elles rencontrent des difficultés à contourner leur propre gouvernement et à rechercher des alliés internationaux pour amener une pression externe afin de supporter la situation de la société civile puisque les pays et les agences intergouvernamentales ont surtout préféré soutenir le gouvernement rwandais post-génocide. ${ }^{16}$

Encore une fois, ce n'est pas une nouveauté post-conflit, des chercheurs indiquent que même si la société civile rwandaise s'est développée pendant les années 1990, ce secteur n'était péniblement indépendant de l'état et il reproduisait les divisions trouvées dans la société. ${ }^{17}$ De la même manière, à cette époque-là, l'insertion de la démocratie ne signifiait pas le dialogue mais plutôt une source utilisée par les élites pour instrumentaliser les masses en exacerbant les clivages horizontaux dans la société afin d'assurer le soutien populaire à la poursuite de leurs propres objectifs. ${ }^{18}$ En rappelant l'enseignement de Jacques Sémelin « l'intensité des passions qui peut mener au génocide dans ces circonstances n'est ni naturelle ni culturelle, mais le résultat des manipulations

\footnotetext{
15 Waldorf, Lars op. cit.. p. 58

${ }^{16}$ Gready, Paul, Beyond "You're with us or against us": Civil Society and Policymaking in Post-Genocide Rwanda" p.92

17 P, Uvin "Prejudice, crisis and genocide in Rwanda" African Studies Review, 40 (2), 1997, p.101 In. SilvaLeander p.1605

18 Silva-Leander, Sebastian, On the Danger and Necessity of Democratisation: trade-offs between shortterm stability and long-term peace in post-genocide Rwanda, Third World Quarterly, vol. 29, No. 8, 2008, p.1605
} 
opérées par les élites, le résultat de la haine fabriquée. "19 À l'égard de ces arguments, les actes du FPR sont rapprochables si on considère les résultats des manipulations, mais non pas leurs justificatifs. Le but de construire une stabilité était atteinte sur ces aspects de l'ingénierie institutionnelle discutés à l'instant, mais ça n'est pas suffisant pour favoriser une paix durable.

\section{La construction de l'histoire officielle et de l'unité nationale}

$\mathrm{Au}$ moment du règlement du conflit, le FPR a gagné un victoire militaire sans aucune aide de la communauté internationale ; au contraire, au Rwanda les casques bleus des Nations Unies et les pays occidentaux étaient vus comme contributeurs directs ou indirects à la violence génocidaire. En 2004 la "Commission Parlementaire Ad Hoc à Analyser Profondément les Massacres Perpétrés à Ginkongoro, l'idéologie du génocide, et à ceux qui le propage partout au Rwanda" a critiqué la communauté internationale pour «semer la division dans la population Rwandaise » il a dénoncé à la BBC, Voice of America, et une multitude d'Églises Chrétiennes pour avoir diffusé l'idéologie du génocide. ${ }^{20}$ À l'égard du discours officiel, le Président Kagame a réagi à un communiqué de l'Union Européenne qui exprimait sa préoccupation devant l'idéologie du génocide, dans un entretien au New Times, un journal rwandais, en disant : «Ce qu'on doit se demander c'est si ce que nous faisons pour le pays est bon ou mauvais, et nous serions prêt pour l'expliquer ». ${ }^{21}$ Le choix du FPR de mener ce type de politique et sa réaction au souci des européens de faire partie de sa multitude de mesures d'ingénierie sociale, son fin majeur, la sécurité, est louable, mais ses résultats sont troublants. Le rôle du FPR dans la suppression et cooptation des médias et des critiques des journalistes, en faisant du recours à l'harcèlement et à l'intimidation si nécessaire, est souligné chez Lars Waldorf dans l'article intitulé « Instrumentalizing Genocide».

Le terme « instrumentalisation du génocide » fait appel au langage accusatoire du gouvernement rwandais envers les critiques Hutus qui a changé et évolué à partir de 2001. La Consitution de 2003 était modifiée, remplaçant "génocide" par « le génocide tutsi de $1994 . »^{22}$ Dans une conférence à Kigali en 2008 un officiel du gouvernement a repris doucement un membre de l'audience pour avoir utilisé le terme "génocide

\footnotetext{
${ }^{19}$ Sémelin, Jacques, op. cit

${ }^{20}$ Commission Parlementaire 2004

21 New Times 2004 In.Waldorf, Lars, Instrumentalizing Genocide “Genocide Ideology” p.53

22 (RoR 2003, arts. 51 and 179)
} 
rwandais" en rappelant que le nouveau terme était "génocide tutsi". ${ }^{23}$ Alors, le gouvernement rwandais s'occupe de criminaliser les actes de "révisionnisme, négationnisme et banalisation du génocide", Président Kagame explique que : «Ceux qui ont des interprétations divergentes à propos de comment et pourquoi le génocide est survenu sont des révisionnistes et ou promoteurs de la théorie du double génocide. Ça, comme nous le savons, est une autre phase du génocide. " ${ }^{24}$ Dans ce processus de victimisation tutsi, la formule " détruire le "eux" pour sauver le "nous"» est déguisé derrière l'idéologie du génocide, atténué par la négation de toute identité comme nous le verrons, mais toujours présent.

Cette séparation par un seuil assez fragile est alarmant, puisque les périodes de crise et de bouleversement durant lesquelles les dirigeants cherchent à redéfinir le « nous » et le « eux »; se chevauchent avec leur manière de transformer l'anxiété en une intense crainte de l'autre en reconstituant les communautés politiques. Le gouvernement utilise le passé traumatique et de l'histoire de manipulation des identités ethniques pour valider ses politiques de criminalisation et stigmatisation de toutes références à l'ethnicité. L'Amnistie Internationale, dans un rapport de 2010 a designé ce phénomène par « chilling effect » en rappelant que ces pratiques ne contribuent pas à instiller la confiance au système de justice, mais bien au contraire. Bref, la Loi de l'Idéologie du Génocide de 2008 a détérioré les efforts vers la création des conditions qui encouragera les réfugiés rwandais à rentrer dans leur pays.

C'est clair que la priorité du FPR (Front patriotique du Rwanda) est d'atteindre l'unité nationale, ça justifie leur mesure de remplacer les identités ethniques par une identité Rwandaise nationale, la seule identité acceptable pour un citoyen Rwandais. Le centre dans une citoyenneté contemporaine plutôt que dans les ethnicités historiquement construites dissout les différences existantes et permet au gouvernement d'incriminer ceux qui défient leur politique étatique, taxés de menaces contre l'unité nationale et la sécurité. Par contre, en ce qui concerne la loi de l'idéologie du génocide, la logique ethnique est vivace au coeur du discours officiel : tout Hutu est suspect puisque son ethnie s'est rendue coupable du génocide. L'histoire se répète : « La révolution de 1959 était limitée à une inversion et une vengeance... maintenant la minorité, pas la majorité...était exclue, mais le monopole ethnique continuait d'être le

${ }^{23}$ Exemple chez Waldorf, p.56

${ }^{24}$ President Kagame 2009 In. Waldorf. p. 57 
principe organisateur de l'état». ${ }^{25}$ C'est une déformation de la conception d'Etat weberien, parce qu'au Rwanda, le monopole n'est pas de la violence physique, mais plutôt de la violence structurelle et symbolique avec la mainmise de l'unité nationale et le principe fondateur de la (non)ethnicité. Il faut qu'on se souvienne de la manière dont, pendant le génocide, les Tutsis étaient ciblés sans aucune considération d'âge, de genre, d'éducation, simplement parce qu'ils étaient assimilés comme ennemis ; ce processus chez Straus s'appelle « catégorisation éthnique collective ». Dans ce contexte, le principe du rejet sélectif aux ethnicités éveille la méfiance à cause de son caractère politique, autoritaire et artificiel, même si allégué comme une façon de promouvoir l'unité nationale au Rwanda post-conflict.

Un autre problème soulevé est celui des personnes multi-positionnées, puisque quand on efface leurs trajectoires, on abandonne l'idée que les individus sont pluriels. Hellen Hintjes souligne que même les divisions de genre sont lentement écartées en présentant les témoignages de personnes qui sont décatégorisées à cause de l'incompatibilité entre ses héritages mixtes et leurs identités politiques polarisés. Ça confirme comment forger de nouvelles identités normalement, échoue si les groupes partagent une histoire de violence intercommunale substantielle À partir d'une analyse de l'autorité imposée du haut jusqu'en bas l'auteur parle du phénomène de « déracialisation » qui a empêché l'émergence potentielle des formes plus complexes d'identité politique. ${ }^{26}$ Également, Danielle Beswick considère le cas de la minorité Batwa, encore plus effacé depuis cette stratégie étatique. ${ }^{27}$ Le processus de detruire les ethnicités existantes et construire un autre, homogène, n'est pas négocié, mais imposé, il présuppose l'existence d'une majorité qui assimile les minorités, et non pas la création d'une nouvelle identité commune. C'est en conséquence, une opération réalisée par l'élite, telle que celle observée dans la session précédente.

Sous la fabrication de l'histoire officielle, l'exemple de la politique d'exhiber les cadavres initié par le gouvernement invoque une façon de mémorisation que n'a pas de rapport avec la tradition funéraire du peuple en considérant que « les rwandais ont horreur des cadavres ». Des survivants ont regretté que de telles cérémonies

\footnotetext{
25 Silva-Lander, Sebastian op. cit p. p.1604

${ }^{26}$ Hintjens, Helen, Post-genocide identity politics in Rwanda, Ethnicities, vol. 8 (1), 2008, p.27

27 Beswick, Danielle, Democracy, identity and the politics of exclusion in post-genocide Rwanda : the case of the Batwa, Democratization, vol. 18, No.2, April 2011, pp.490-511
} 
contribuent à réintroduire les participants dans la violence plutôt que de les aider à la dépasser :28

« Comment parler de la réconciliation si l'exposition des squelettes consiste à rappeler à certains que les autres ont tué les leurs? C'est maintenir les uns dans une position de culpabilité éternelle, ce n'est pas seulement de raviver la haine chez les autres, c'est de ne pas permettre à leurs plaies de cicatriser. La haine grandissante d'un côté, de l'autre la peur permanente.» 29

Également, en ce qui concerne les victimes, la commémoration publique est sélective parce qu'elle ne retient que certaines d'entre elles, ou encore les hiérarchise, ce qui revient à exercer une violence symbolique. Prenons l'exemple de la pastorale du deuil : même si elle devait comprendre toutes les victimes, ceux du génocide mais aussi les victimes tuées par l'armée du FPR, et pourtant la commission n'ignorait pas que la mention des « autres massacres » violait la loi du silence. C'est-à-dire que cette histoire officielle, qui est construite à partir de la commémoration du désastre, tend à interdire, supplanter ou refouler, selon les situations, une connaissance libre et plurielle de ce qui s'est passé.

Avec les Gacacas c'est pareil, elles n'ont pas résolu toutes les causes du génocide, parce que ce n'était pas conçu comme une solution pour les litiges mais simplemement un tribunal et même les tribunaux ne peuvent pas mettre un terme définitif aux conflits. En plus, les Gacacas souffrent, dès le départ, de deux handicaps : ils ne concernent pas les Hutus trop lourdement impliqués et ils ne concernent pas non plus les crimes de guerre commis par les soldats du FPR, non catégorisés comme génocide et relevant des juridictions militaires classiques lorsqu'ils sont poursuivis. ${ }^{30}$ Cette initiative prise par le gouvrnement rwandais mérite d'être remis en question en ce qui concerne le modèle «Vérité et réconciliation » proposé :

«Si l'on veut vraiment résoudre les conflits entre Rwandais, il faut le faire sur un pied d'égalité. Il ne faut pas dire que seuls les Hutus devraient s'adresser aux Tutsis pour leur demander pardon. Par exemple, pendant la Gacaca en prison, on nous a dit d'avouer et de plaider coupable et de demander pardon à ceux à qui nous avons causé du tort. Or il se trouve qu'il y avait des prisonniers qui n'avaient pas commis le moindre crime et qui étaient emprisonnés injustement. Et donc ceci a été dit, mais aucune mention n'a été faite de ces autres individus qui ont fait emprisonner des gens injustement. Pourquoi ne pas demander aux rescapés de demander pardon aux prisonniers innocents qui ont été relâchés, tout comme les coupables l'ont fait auprès des familles victimes par exemple ? À mon avis, c'est ce qu'il faudrait faire si nous voulons parvenir à la

\footnotetext{
28 Vidal, Claudine, « La commemoration do génocide au Rwanda : Violence symbolique, mémorisation forcée et histoire oficielle »

${ }^{29}$ R. M., 10 novembre 200, Bruxelles, lettre à l'auteur In Vidal, Claudine, op. cit. p. 586

${ }^{30}$ Paillet, Paule, Après le génocide: justice au Rwanda, Informations sociales, 2005/7 n 127, p. 25
} 
réconciliation entre tous les Rwandais. Sinon la réconciliation ne se fera qu’à sens unique. $»^{31}$

Alors, une réconciliation à sens unique a été faisable à court terme mais ce n'est pas la solution la plus désirable à long terme

\section{Conclusion}

Après la fin des Gacacas, ce qui pose problème, c'est le fait que le gouvernement les a remplacées par sa loi contre l'idéologie du génocide, qui de même contribue à la dénonciation fondée sur des conflits politiques ou personnels. Afin de guérir des blessures individuelles, le 'truth telling' normalement éconduit le mythe que les groupes ethniques adversaires portent une responsabilité collective pour les crimes. L'enjeu à propos du modèle établit au Rwanda post-conflict c'est son imperméabilité aux individus. Parce que même si la réhabilitation individuelle est un processus complexe, et si l'ordre social peut être réparé sans que les victimes en tant qu'individus soient guéries, ce qu'on observe au Rwanda c'est une erreur par rapport au concept de « victimes », qui est devenu synonyme de Tutsi, et le concept de "génocidaires », qui est devenu synonyme de Hutu.

Au-delà de la critique à ce modèle de réconciliation à sens unique implanté au Rwanda sous l'ingénierie sociale menée par le FPR, l'objectif de cette étude était de démontrer comment le discours de l'unité nationale est faible et sélectif. Alors, la solution des affaires tel qu'elle était pensée atteint les objectifs à court terme, paix négative, mais ne constitue pas une solution permanente, paix durable ou paix positive, qu'on aimerait établir après les violences massives. Les politiques de paix ne peuvent pas être classées en bonnes ou mauvaises, comme Paul Kagame l'a remarqué, pour les évaluer il faut tout d'abord abandonner ces notions maniquéistes et craver des modèles et des explications plus élaborés. Le contexte, la situation politique, l'histoire, la culture, doivent être prises en compte.

"Il reste que les autorités détruisent ce que le travail de vérité aurait pu avoir de positif : parce qu'à la
violence $\quad$ passée,

\footnotetext{
31 Penal reform International, Huit ans après...Le point sur le monitoring de la gacaca au Rwanda, Prisonnier libéré, 2003.

${ }^{32}$ Vidal, Claudine, op. cit. p. 581
} 
Bibliographie

Amnesty International, Safer to Stay Silent: The Chilling Effect of Rwanda's Laws on 'Genocide Ideology' and 'Sectarianism', Amnesty International Publicaitons, 2010

BESWICK, Danielle, Democracy, identity and the politics of exclusion in post-genocide Rwanda : the case of the Batwa, Democratization, vol. 18, No.2, April 2011, pp.490-511

CLARK, Janine, Learning from the Past: Three Lessons from the Rwandan Genocide, African Studies, 68, 1, April 2009

GALTUNG, Johan, Violence, peace, and peace research, Journal of Peace Research, vol.6, no.3, 1969, pp.167-191

GREADY, Paul, Beyond "You're with us or against us": civil society and Policymaking in Post-Genocide Rwanda"

HINTJENS, Helen, Post-genocide identity politics in Rwanda, Ethnicities, vol. 8 (1), 2008

Hirondelle News Agency "Carla Del Ponte tells of her attempts to investigate RPF in her new book" 2 avril 2008. Disponible sur: http://www.hirondellenews.com/ictrrwanda/407-collaboration-with-states/collaboration-with-states-rwanda/21710-enen-020408-ictrrwanda-carla-del-ponte-tells-of-her-attempts-to-investigate-rpf-in-hernew-book1076110761

MENDELOFF, David, Truth-Seeking, Truth-Telling, and. Postconflict Peacebuilding: Curb the Enthusiasm?, International Studies Review, Vol. 6, No. 3, September 2004, pp. 355380

OLESEN, Thomas, Global Injustice Memories: The 1994 Rwanda Genocide, Global Injustice Memories, International Political Sociology, 6, 2012, pp.373-389

PAILLET, Paule, Après le génocide: justice au Rwanda, Informations sociales, 2005/7 n 127, p. 25-26.

Penal reform International, Huit ans après...Le point sur le monitoring de la gacaca au Rwanda, 2010, Disponible sur: $\underline{\text { http://www.penalreform.org/wp- }}$ content/uploads/2013/05/WEB-french-gacaca-rwanda-6-1.pdf Accès en 25/10 
SAMSET, Ingrid, Building a Repressive Peace :The Case of Post-Genocide Rwanda, Journal of Intervention and Statebuilding, vol.5, No.3, September 2011, p.265-283

SEMELIN, Jacques. Pour sortir de la violence, Paris, Les Editions ouvrières, 1983, p.16

STRAUS, Scott, WALDORF, Lars, Remaking Rwanda : State-Building and Human Rights after Mass Violence, The University of Wisconsin Press, Wisconsin, 2011

SILVA-LEANDER, Sebastian, On the Danger and Necessity of Democratisation: trade-offs between short-term stability and long-term peace in post-genocide Rwanda, Third World Quarterly, vol. 29, No. 8, 2008, pp. 1601-1620

VIDAL, Claudine, La commemoration do génocide au Rwanda: Violence symbolique, mémorisation forcée et histoire oficielle, Cahiers d'Études africaines, XLIV (3), 175, 2004, pp.575-592

WALDORF, Lars, L'Instrumentalisation du Génocide : The RPF's campaign against « Genocide Ideology » In. Remaking Rwanda : State Building and Human Rights after Mass Violence 